06

\title{
Сверхбыстрое спекание оксидных керамических материалов при микроволновом нагреве
}

\author{
() Ю.В. Быков, ${ }^{1}$ С.В. Егоров, ${ }^{1}$ А.Г. Еремеев, ${ }^{1}$ И.В. Плотников, ${ }^{1}$ К.И. Рыбаков, ${ }^{1,2, \Phi}$ \\ А.А. Сорокин, ${ }^{1}$ В.В. Холопцев ${ }^{1}$ \\ ${ }^{1}$ Институт прикладной физики РАН, \\ 603155 Нижний Новгород, Россия \\ ${ }^{2}$ Нижегородский государственный университет им. Н.И. Лобачевского, \\ 603000 Нижний Новгород, Россия \\ ฯ e-mail: rybakov@appl.sci-nnov.ru
}

(Поступило в Редакцию 23 июня 2017 г.)

\begin{abstract}
Представлены результаты исследования эффекта сверхбыстрого спекания, наблюдающегося при нагреве компактированных порошковых материалов интенсивным микроволновым излучением. Образцы керамических материалов $\mathrm{Y}_{2} \mathrm{O}_{3}, \mathrm{MgAl}_{2} \mathrm{O}_{4}$ и $\mathrm{Yb}:(\mathrm{LaO})_{2} \mathrm{O}_{3}$ были спечены до плотности более $98-99 \%$ от теоретического значения за время 0.5-5 min без изотермической выдержки. При этом удельная мощность микроволнового излучения, поглощаемая в объеме образцов, составляла $20-400 \mathrm{~W} / \mathrm{cm}^{3}$. На основании анализа экспериментальных данных (мощность микроволнового излучения, скорости нагрева и остывания) и исследований микроструктуры полученных материалов предложен механизм сверхбыстрого спекания, основанный на развитии тепловой неустойчивости и размягчении (плавлении) границ зерен. Предложенный механизм объясняет также эффект сверхбыстрого спекания (flash sintering), наблюдаемый при приложении к образцам постоянного или низкочастотного переменного напряжения. Применение микроволнового нагрева позволяет реализовать процессы сверхбыстрого спекания без необходимости использования электродов для обеспечения энерговклада в спекаемые изделия.
\end{abstract}

DOI: 10.21883/JTF.2018.03.45598.2398

\section{Введение}

В последнее время активно развиваются методы спекания керамических, композиционных и металлических материалов с применением электрических токов и электрических полей. Ускоренное уплотнение порошковых компактов наблюдается при использовании таких методов, как спекание с применением внешних полей (Field Assisted Sintering Techniques, FAST), спекание импульсными токами (Pulsed Electric Current Sintering, PECS), электроискровое плазменное спекание (Spark Plasma Sintering, SPS) и микроволновое спекание. Ocновные особенности этих методов в применении к широкому классу керамических материалов рассмотрены в обзорах [1-3]. Их общей отличительной особенностью является значительное сокращение времени, необходимого для консолидации порошковых материалов, по сравнению с традиционными технологиями спекания.

Еще большее сокращение времени процесса наблюдается при спекании методом, получившим название flash sintering [4,5]. В этом методе к образцу, нагреваемому сторонними источниками тепла, например в печи резистивного нагрева, прикладывают постоянное или низкочастотное переменное напряжение. Эффект сверхбыстрого (зачастую за несколько секунд) спекания материала до плотности, близкой к теоретической, возникает при определенном сочетании температуры и величины удельной мощности, выделяемой в образце вследствие протекания через него электрического тока. Механизмы, ответственные за данный эффект сверхбыстрого спекания, к настоящему времени не установлены и являются предметом дискуссий.

Очевидно, что протекание тока через образец приводит к его дополнительному нагреву. При определенном соотношении между удельной мощностью объемного нагрева джоулевым источником тепла и удельной мощностью потерь тепла с поверхности образца может развиться тепловая (перегревная) неустойчивость. Неустойчивость наблюдается при нагреве материалов, в которых эффективная электрическая проводимость растет с температурой, что создает нелинейную положительную обратную связь [6].

В настоящее время общепризнано, что экспериментально наблюдаемые два одновременно протекающих события - увеличение электрической проводимости, регистрируемой по нарастанию тока, и сверхбыстрое уплотнение материала - собственно, flash sintering являются следствием развития тепловой неустойчивости.

Сам по себе экспоненциальный рост проводимости никак не проясняет причины резкого ускорения спекания. Как следует из оценок [7], увеличение температуры в объеме спекаемых образцов при развитии тепловой неустойчивости может достигать $800^{\circ} \mathrm{C}$ по сравнению с температурой, предшествующей началу спекания. Однако, согласно [7], такого увеличения температуры недостаточно для того, чтобы объяснить наблюдающееся сверхбыстрое спекание возрастанием скоростей терми- 
чески активируемых процессов твердофазного диффузионного массопереноса.

Явление тепловой неустойчивости хорошо известно в практике микроволнового нагрева материалов [8,9]. Микроволновый нагрев материалов осуществляется за счет поглощения в них энергии электромагнитного излучения. Коэффициенты поглощения излучения во многих материалах, в том числе в различных керамических композициях, возрастают с температурой. Если при нагреве параметр $\beta=\left(d p_{\text {abs }} / d T\right)\left(T / p_{\text {abs }}\right)$ (где $p_{\text {abs }}$ - мощность, поглощаемая в единице объема образца, $T$ - температура) превышает определенное критическое значение, в образце развивается тепловая неустойчивость [10]. Как правило, возможность неконтролируемого развития тепловой неустойчивости рассматривается в качестве одного из основных недостатков использования микроволнового нагрева для высокотемпературной обработки материалов.

В настоящей работе показано, что управление двумя основными параметрами - удельной поглощенной в образце мощностью и мощностью тепловых потерь - и использование быстрого автоматического контроля микроволновой мощности позволяет реализовать эффект сверхбыстрого спекания при микроволновом нагреве. Приведены результаты экспериментальных исследований сверхбыстрого спекания оксидных керамических материалов $\mathrm{Yb}:(\mathrm{LaY})_{2} \mathrm{O}_{3}, \mathrm{MgAl}_{2} \mathrm{O}_{4}$ и $\mathrm{Y}_{2} \mathrm{O}_{3}$ до плотности, близкой к теоретической, за время, не превышающее единиц минут. На основе анализа факторов, существенных для реализации эффекта сверхбыстрого микроволнового спекания, и результатов исследования микроструктуры спеченных материалов предложен механизм уплотнения в условиях развития в спекаемом материале тепловой неустойчивости.

\section{Материалы и методы}

В экспериментах использовались образцы составов $\mathrm{Y}_{2} \mathrm{O}_{3}, \mathrm{MgAl}_{2} \mathrm{O}_{4}$ и 5 at.\% Yb: $\left(\mathrm{La}_{0.1} \mathrm{Y}_{0.9}\right)_{2} \mathrm{O}_{3}$, изготовленные из синтезированных в Институте химии высокочистых веществ РАН (Нижний Новгород) наноразмерных порошков. Определенные методом Брунауэра-ЭмметаТеллера (ВET) размеры частиц порошков составляли $130\left(\mathrm{Y}_{2} \mathrm{O}_{3}\right), 10\left(\mathrm{MgAl}_{2} \mathrm{O}_{4}\right)$ и $140 \mathrm{~nm} \quad\left(\mathrm{Yb}:(\mathrm{LaY})_{2} \mathrm{O}_{3}\right)$. Холодным одноосным прессованием при давлении 150-400 МРа изготавливались образцы в форме дисков диаметром 10-15 mm и толщиной порядка $2.5 \mathrm{~mm}$. Относительные плотности изготовленных образцов равнялись $0.42\left(\mathrm{Y}_{2} \mathrm{O}_{3}\right), 0.38\left(\mathrm{MgAl}_{2} \mathrm{O}_{4}\right)$ и $0.52\left(\mathrm{Yb}:(\mathrm{LaY})_{2} \mathrm{O}_{3}\right)$ от соответствующих теоретических значений.

Образцы нагревали в рабочей камере гиротронного комплекса мощностью до $6 \mathrm{~kW}$ на частоте $24 \mathrm{GHz}$, оснащенного компьютерной системой управления мощностью [11]. Образцы помещались в центре цилиндрического кварцевого тигля диаметром $100 \mathrm{~mm}$ и высотой $100 \mathrm{~mm}$. Для теплоизоляции образцов тигель за- полняли крупнозернистыми порошками оксида иттрия (при спекании образцов $\mathrm{Yb}:(\mathrm{LaY})_{2} \mathrm{O}_{3}$ и $\left.\mathrm{Y}_{2} \mathrm{O}_{3}\right)$ или оксида алюминия (при спекании образцов $\mathrm{MgAl}_{2} \mathrm{O}_{4}$ ). Температуру измеряли $\mathrm{Pt}-\mathrm{Pt}-\mathrm{Rh}$-термопарой, головка которой касалась образца в центре нижней поверхности дисков. Для удаления остатков использовавшейся при компактировании связки образцы нагревали в атмосфере воздуха со скоростью $10^{\circ} \mathrm{C} / \mathrm{min}$ до температуры $800^{\circ} \mathrm{C}$. По окончании данной предварительной стадии нагрева рабочую камеру откачивали до давления порядка 2-5 Pa и выполняли высокоскоростную стадию нагрева.

Образцы нагревали от $T_{0}=800^{\circ} \mathrm{C}$ до заданной максимальной температуры $T_{m}$ с постоянной скоростью 50, 100, 150 либо $200^{\circ} \mathrm{C} / \mathrm{min}$. Микроволновая мощность автоматически отключалась при достижении заданного значения $T_{m}$, после чего образец остывал вместе с окружающей его теплоизолирующей засыпкой.

Плотность спеченных образцов определяли методом взвешивания в дистиллированной воде с точностью $\pm 0.01 \mathrm{~g} / \mathrm{cm}^{3}$. Микроструктуру спеченных образцов исследовали с помощью сканирующего электронного микроскопа JEOL JSM-6390 LV. Распределения химических элементов анализировали методом энергодисперсной спектрометрии, используя детектор JEOL EX-54175, совмещенный с электронным микроскопом. Зеренную структуру исследовали с помощью атомно-силового микроскопа Smena NT-MDT. Фазовый состав спеченных образцов анализировали с использованием рентгеновского дифрактометра Rigaku Ultima IV.

\section{Результаты и обсуждение}

В исследованиях процесса сверхбыстрого спекания при приложении к образцам постоянного(переменного) напряжения их уплотнение регистрируется дилатометром $[4,5]$. Одновременно с быстрым уменьшением размеров образцов происходит изменение электрической проводимости, которое сопровождается резким ростом протекающего через них тока и увеличением выделяемой в них плотности мощности $p=j U / L$, где $j-$ плотность тока, $U$ - приложенное к образцу напряжение, $L-$ длина спекаемой части образца. При микроволновом спекании отсутствует возможность непосредственного измерения удельной поглощаемой в образце плотности мощности. Однако сопутствующий сверхбыстрому спеканию эффект изменения эффективной высокочастотной проводимости $\sigma$ может быть экспериментально зарегистрирован по поведению автоматически регулируемой в процессе нагрева мощности $P_{\text {input, }}$ поступающей в рабочую камеру. Для этого необходимо принять во внимание рассмотренную ниже связь между удельной поглощенной в образце мощностью

$$
p_{\mathrm{abs}}=\sigma E_{s}^{2}
$$

(где $E_{s}$ - напряженность электрического поля в образце) и мощностью $P_{\text {input }}$. 
Рабочая камера гиротронного комплекса для нагрева материалов электромагнитным излучением миллиметрового диапазона представляет собой ненастренный многомодовый резонатор диаметром порядка $50 \mathrm{~cm}$ и длиной около $60 \mathrm{~cm}$. Отношение его объема $V$ к кубу длины волны излучения $\lambda$, равной $1.25 \mathrm{~cm}$, составляет порядка $10^{5}$. В таком резонаторе распределение электромагнитного поля по объему является квазиоднородным, поскольку поле представляет собой суперпозицию большого количества одновременно возбуждаемых электромагнитных мод [12,13]. Добротность $Q$ резонатора такого типа определяется главным образом омическими потерями в его стенках. (Потери в образце малы в силу его сравнительно небольших размеров, а потери на связь с подводящим трактом малы из-за сравнительно небольшой площади входного отверстия резонатора.) Величина входящей мощности $P_{\text {input }}$ определяет запасенную в резонаторе энергию $W$ электромагнитного поля [14]:

$$
W=\varepsilon_{0} \int E^{2} d V=\frac{Q}{\omega} P_{\text {input }},
$$

где $\varepsilon_{0}-$ электрическая постоянная, $E-$ напряженность электрического поля в резонаторе, $\omega-$ циклическая частота микроволнового излучения. Напряженность электрического поля $E_{s}$ в помещенном в резонатор образце небольших размеров из материала с диэлектрической проницаемостью $\varepsilon \sim 5-15$ близка к напряженности электрического поля в резонаторе вне образца: $E_{s} \approx E$. Тогда, используя (1) и (2), легко получить соотношение между мощностью $P_{\text {input }}$ и мощностью $p_{\text {abs }}$, поглощаемой в единице объема образца:

$$
p_{\mathrm{abs}} \approx \frac{Q}{\varepsilon_{0} \omega V} \sigma P_{\text {input }} .
$$

Поглощаемая в образце мощность микроволнового излучения расходуется на увеличение его внутренней энергии и на компенсацию потерь тепла при его нагреве. Удельную поглощенную мощность $p_{\text {abs }}$, затрачиваемую для достижения образцом заданной температуры $T_{m}$, легко оценить, исходя из уравнения баланса энергии, в простейшем случае ее однородного распределения в объеме образца и в предположении отсутствия поглощения излучения в материале теплоизоляции. Используя данные о скоростях нагрева и остывания образца непосредственно до и после достижения температуры $T_{m}$ (и отключения микроволновой мощности), имеем

$$
p_{\mathrm{abs}}=\rho C\left(\frac{d T}{d t}\right)_{+}+p_{\mathrm{hl}}, \quad p_{\mathrm{hl}}=\rho C\left|\left(\frac{d T}{d t}\right)_{-}\right|,
$$

где $\rho$ - плотность, $C-$ удельная теплоемкость материала образца, $p_{\mathrm{hl}}-$ мощность тепловых потерь, $(d T / d t)_{+}$и $\left|(d T / d t)_{-}\right|$- скорости нагрева и охлаждения непосредственно до и после момента отключения микроволновой мощности соответственно.

Мощность тепловых потерь $p_{\mathrm{hl}}$ при температуре порядка и свыше $1000^{\circ} \mathrm{C}$ определяется в основном
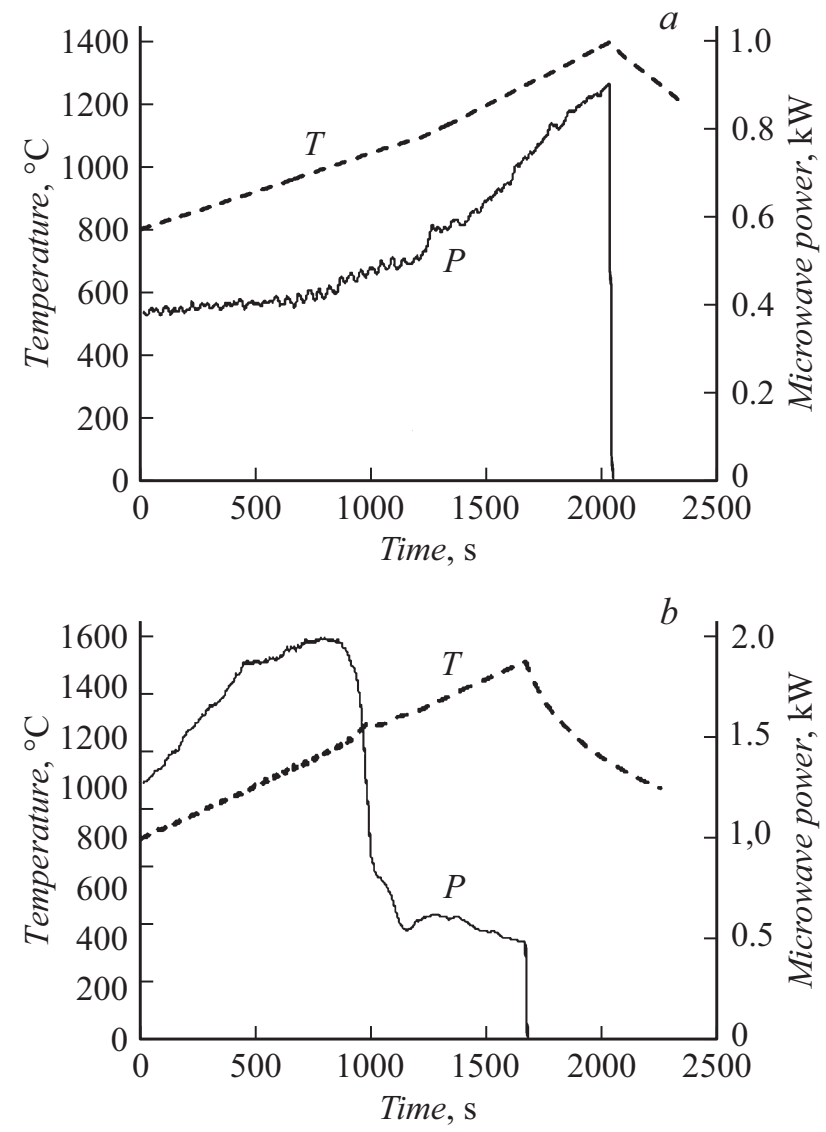

Рис. 1. Температура и вводимая в рабочую камеру гиротронного комплекса микроволновая мощность на высокотемпературной стадии спекания образцов керамики $\mathrm{Yb}:(\mathrm{LaY})_{2} \mathrm{O}_{3}$ в зависимости от времени: $a-$ скорость нагрева $15^{\circ} \mathrm{C} / \mathrm{min}$, максимальная температура $1400^{\circ} \mathrm{C} ; b-$ скорость нагрева $50^{\circ} \mathrm{C} / \mathrm{min}$, максимальная температура $1500^{\circ} \mathrm{C}$.

тепловым излучением и возрастает пропорционально $T^{4}$ по мере увеличения температуры образца. Обычно при микроволновом нагреве с невысокой скоростью в условиях эффективной теплоизоляции мощность $p_{\text {abs, }}$, поглощаемая в единице объема образца, и связанная с ней соотношением (3) мощность $P_{\text {input }}$ монотонно возрастают по мере роста температуры, как показано на рис. 1, a (мы не рассматриваем случаи, когда в материале образца происходят фазовые превращения, сопровождающиеся изменениями его внутренней энергии). Эффективная высокочастотная электрическая проводимость $\sigma$ для большинства материалов также увеличивается с ростом температуры. Однако при малой скорости нагрева в условиях эффективной теплоизоляции и, следовательно, невысокой величине $P_{\text {input }}$ параметр $\beta=\left(d p_{\text {abs }} / d T\right)\left(T / p_{\text {abs }}\right)$ не превышает критического значения, и тепловая неустойчивость не возникает.

Иное поведение наблюдается при высокой скорости нагрева спекаемого образца в условиях слабой теплоизоляции, т. е. при сравнительно большой требуемой для нагрева удельной поглощенной мощности $p_{\text {abs }}($ рис. $1, b)$. 
Входная мощность $P_{\text {input }}$, автоматически регулируемая в соответствии с заданным температурно-временным режимом нагрева, возрастает до момента достижения некоторого значения температуры $T^{*}$ и затем резко уменьшается. Несмотря на уменьшение $P_{\text {input, }}$ температура образца продолжает увеличиваться с заданной постоянной скоростью, что, как следует из уравнения баланса энергии (4), свидетельствует о дальнейшем увеличении мощности $p_{\text {abs, }}$, поглощаемой в единице объема образца. В соответствии с (3) наблюдаемое автоматическое снижение входящей мощности в ходе нагрева с постоянной скоростью может быть вызвано только резким возрастанием эффективной высокочастотной электрической проводимости материала образца, являющимся следствием развития тепловой неустойчивости. Увеличение проводимости обеспечивает продолжение возрастания удельной поглощаемой мощности $p_{\text {abs, }}$ необходимое для поддержания постоянной скорости нагрева, несмотря на снижение величины $E^{2}$, пропорциональной подводимой мощности.

Резкий рост эффективной проводимости связан с изменениями в фазовом состоянии материала образца - a именно началом формирования в нем квазижидкой фазы, величина электрической проводимости которой намного выше, чем у твердого материала зерен. Возрастание при этом поглощаемой микроволновой мощности приводит к кратковременному отклонению фактического хода температуры образца от предписанного линейного роста (рис. $1, b$ ), что свидетельствует о развитии тепловой неустойчивости, которое в дальнейшем компенсируется системой автоматического управления микроволновой мощностью.

В таблице приведены некоторые результаты экспериментов по сверхбыстрому микроволновому спеканию керамических образцов указанных составов $[15,16]$. В ходе экспериментов определялись скорости нагрева $(d T / d t)_{+}$, условия теплоизоляции (определяющие скорости охлаждения $\left.(d T / d t)_{-}\right)$и на основе результатов пробных тестов задавались такие значения максимальной температуры нагрева $T_{m}$, при которых в ходе нагрева наблюдался бы резкий спад входной мощности $P_{\text {input }}$ и которые бы на $50-100^{\circ} \mathrm{C}$ превышали значение температуры, соответствующее началу спада мощности.

Керамические образцы всех перечисленных составов плотностью более $98 \%$ от соответствующих теоретических значений были получены в процессах без изотермической выдержки при продолжительности стадии нагрева в интервале температур $T_{m}-T_{0}$, не превышающей $10 \mathrm{~min}$. Отметим, что данный временной интервал может рассматриваться как верхняя оценка времени спекания, поскольку температура $T_{0}$ (температура удаления связки) заведомо ниже температуры начала спекания.

Сравнение параметров процесса спекания материалов номинально одного состава, но разного происхождения неправомерно вследствие различия характеристик исходных порошков и методов приготовления порошковых компактов, способных повлиять на их спекаемость. Тем
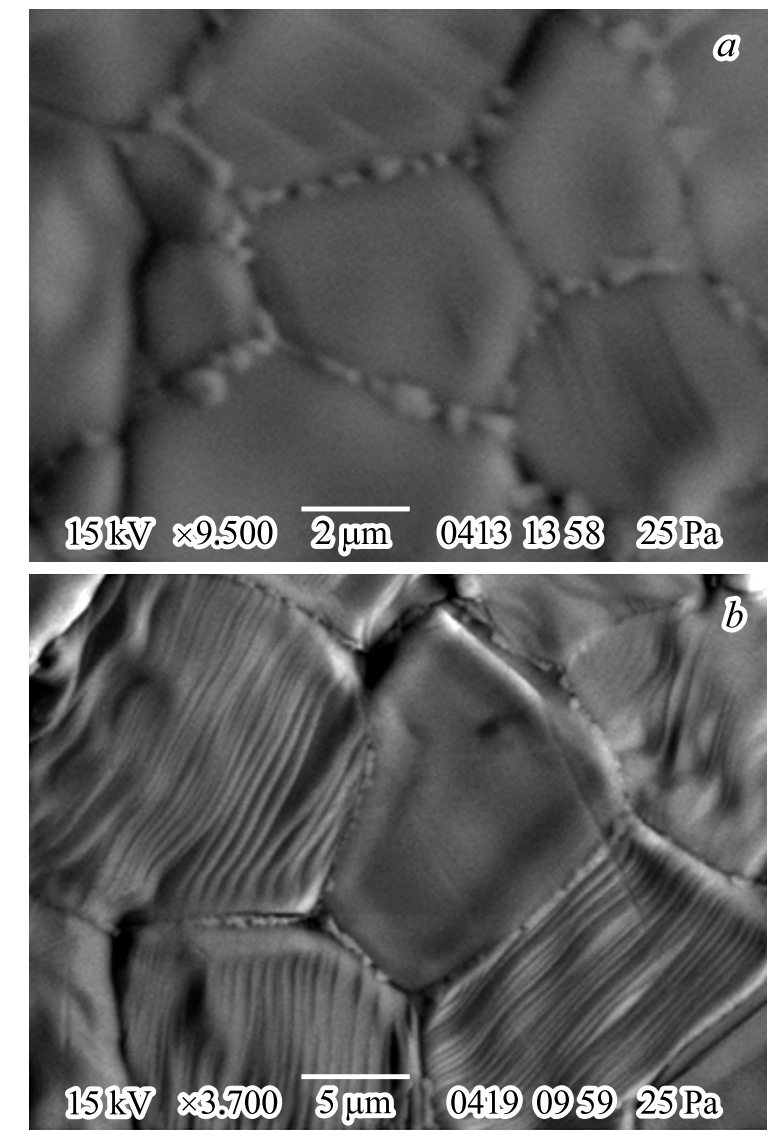

Рис. 2. Электронно-микроскопические изображения микроструктуры образцов керамики $\mathrm{Yb}:\left(\mathrm{La}_{0.1} \mathrm{Y}_{0.9}\right)_{2} \mathrm{O}_{3}$, спеченных в режимах сверхбыстрого микроволнового спекания: $a-$ скорость нагрева $50^{\circ} \mathrm{C} / \mathrm{min}$, максимальная температура $1500^{\circ} \mathrm{C}$; $b$ - скорость нагрева $200^{\circ} \mathrm{C} / \mathrm{min}$, максимальная температуpa $1500^{\circ} \mathrm{C}$.

не менее отметим, что времена спекания, полученные в настоящей работе, на порядки отличаются от значений, характерных для спекания материалов того же состава в традиционных процессах (как правило, порядка нескольких часов). Так, в [17] $\mathrm{MgAl}_{2} \mathrm{O}_{4}$-керамика с относительной плотностью 99\% получена при двухчасовой изотермической выдержке при температуре $1750^{\circ} \mathrm{C}$. Керамика состава $\mathrm{Yb}:(\mathrm{LaY})_{2} \mathrm{O}_{3}$ является одним из перспективных лазерных поликристаллических материалов. Лазерная керамика данного состава изготавливается спеканием в вакуумной печи в атмосфере водорода при температуpe $1650^{\circ} \mathrm{C}$ в течение $50 \mathrm{~h}$ [18]. Методом сверхбыстрого микроволнового спекания высокопрозрачные и, следовательно, практически беспористые образцы керамики были получены при нагреве со скоростью $100^{\circ} \mathrm{C} / \mathrm{min}$ до температуры $1550^{\circ} \mathrm{C}$ без изотермической выдержки [15].

Результаты исследования микроструктуры образцов керамики состава $\mathrm{Yb}$ :(LaY $)_{2} \mathrm{O}_{3}$, спеченных в условиях развития тепловой неустойчивости, подтверждают факт образования квазижидкой фазы в процессе спекания [15]. Так, в образцах, спеченных при относительно невысо- 
Результаты экспериментов по сверхбыстрому микроволновому спеканию керамических образцов

\begin{tabular}{|c|c|c|c|c|c|}
\hline $\begin{array}{c}\text { Максимальная } \\
\text { температура, }{ }^{\circ} \mathrm{C}\end{array}$ & $\begin{array}{c}\text { Скорость нагрева, } \\
{ }^{\circ} \mathrm{C} / \mathrm{min}\end{array}$ & $\begin{array}{c}\text { Скорость остывания, } \\
{ }^{\circ} \mathrm{C} / \mathrm{min}\end{array}$ & $\begin{array}{c}\text { Конечная } \\
\text { плотность, \% }\end{array}$ & $\begin{array}{l}\text { Время нагрева, } \\
\min \end{array}$ & $\begin{array}{c}\text { Удельная } \\
\text { поглощенная } \\
\text { мощность, W/cm } 3\end{array}$ \\
\hline \multicolumn{6}{|c|}{$\mathrm{Yb}:\left(\mathrm{La}_{0.1} \mathrm{Y}_{0.9}\right)_{2} \mathrm{O}_{3}$} \\
\hline 1500 & 100 & 200 & 98.5 & 7.0 & 19 \\
\hline 1500 & 150 & 200 & 98.5 & 4.7 & 22 \\
\hline 1500 & 200 & 200 & 97.2 & 3.5 & 25 \\
\hline \multicolumn{6}{|c|}{$\mathrm{Y}_{2} \mathrm{O}_{3}$} \\
\hline 1600 & 100 & 385 & 98.3 & 8.0 & 40 \\
\hline 1700 & 100 & 420 & 98.1 & 9.0 & 43 \\
\hline \multicolumn{6}{|c|}{$\mathrm{MgAl}_{2} \mathrm{O}_{4}$} \\
\hline 1650 & 100 & 290 & 98.1 & 8.5 & 17.5 \\
\hline 1780 & 150 & 230 & 98.3 & 6.5 & 15 \\
\hline
\end{tabular}

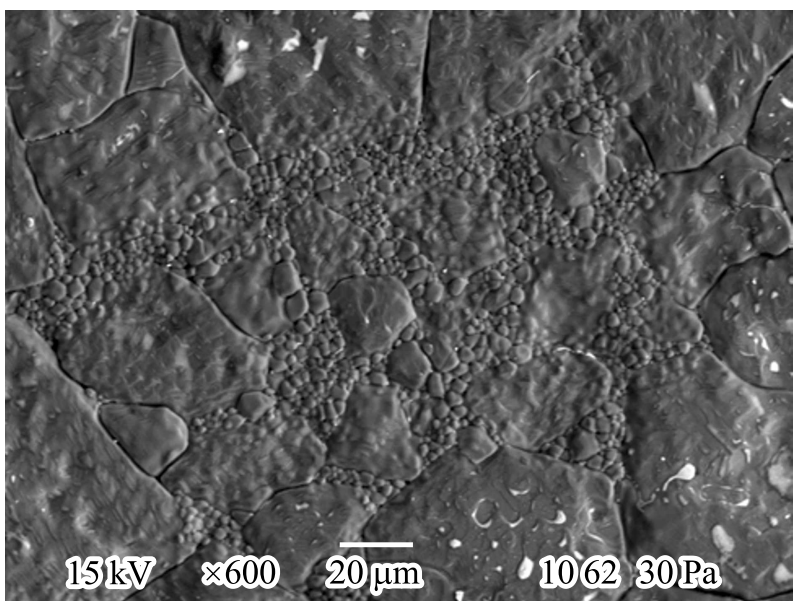

Pис. 3. Электронно-микроскопическое изображение микроструктуры образца шпинели $\mathrm{MgAl}_{2} \mathrm{O}_{4}$ (с добавкой $1 \% \mathrm{Y}_{2} \mathrm{O}_{3}$ ), спеченного в режиме сверхбыстрого микроволнового спекания: скорость нагрева $150^{\circ} \mathrm{C} / \mathrm{min}$, максимальная температуpa $1780^{\circ} \mathrm{C}$.

ких температурах и значениях удельной поглощаемой мощности $\left(p_{\mathrm{abs}} \approx 15 \mathrm{~W} / \mathrm{cm}^{3}\right)$, на границах зерен наблюдаются мелкие сферические каплеобразные образования (рис. 2,a). При увеличении величины удельной поглощаемой мощности $\left(p_{\mathrm{abs}} \approx 23 \mathrm{~W} / \mathrm{cm}^{3}\right)$ общий объем квазижидкой фазы увеличивается, капли сливаются, образуя межзеренные прослойки (рис. 2, $b$ ). Образованию таких прослоек способствует также размягчение более широких областей, прилежащих к границам зерен. Как известно [19], данные явления на границах зерен характерны для спекания керамики с участием жидкой фазы.

Выполненные на образцах состава $\mathrm{Yb}:(\mathrm{LaY})_{2} \mathrm{O}_{3}$ исследования зеренной структуры показали, что кинетика роста зерна в образцах, спеченных при значениях $p_{\text {abs }} \geq 15 \mathrm{~W} / \mathrm{cm}^{3}$, типична для жидкофазного спе- кания и существенно отличается от кинетики роста при твердофазном спекании образцов в аналогичных режимах традиционного нагрева [15]. Ускорение роста зерна типично для спекания керамических материалов с участием жидкой фазы [20]. Результаты исследования микроструктуры образцов $\mathrm{MgAl}_{2} \mathrm{O}_{4}$-шпинели, спеченной в режиме сверхбыстрого нагрева, свидетельствуют о выраженном бимодальном распределении размеров зерна и их аномальном росте (рис. 3), что также типично для процесса жидкофазного спекания [19].

\section{Обсуждение результатов}

На основании анализа полученных экспериментальных результатов предложен следующий механизм сверхбыстрого спекания [16].

При малых размерах зерна (порядка и менее микрона) перенос тепла посредством теплопроводности исключает возможность заметного различия в температурах границы и объема зерен нагреваемого материала [21]. Однако вследствие повышенной концентрации примесей и дефектов в приграничных областях поликристаллических зерен температура плавления в этих областях может существенно отличаться от температуры плавления чистого материала [22]. В результате размягчение поверхности зерен может происходить при существенно более низких температурах, чем плавление в объеме зерен.

При объемном микроволновом нагреве компактированного порошкового образца, сопровождаемом отводом тепла через его поверхность, устанавливается неоднородное распределение температуры с максимумом во внутренней области образца (так называемое инверсное распределение). В том случае, когда температура и удельная поглощаемая мощность достаточно велики, в этой области за счет размягчения и плавления границ 
зерен начинается образование квазижидкой фазы, имеющей существенно более высокую эффективную электрическую проводимость, чем проводимость твердого диэлектрического материала. В свою очередь, это приводит к резкому усилению поглощения микроволновой мощности и развитию тепловой неустойчивости.

Вследствие плавления на границах твердые зерна оказываются окружены квазижидкой фазой со сравнительно низкой вязкостью. За счет растворения твердого материала и интенсивного диффузионного массопереноса через квазижидкую фазу зерна приобретают гладкую округлую форму. Квазижидкая фаза полностью смачивает зерна в силу тождественности их химического состава. Действующие капиллярные силы, величина которых, согласно оценке [16], может составлять десятки МРа, приводят к повороту и проскальзыванию зерен относительно друг друга и, в конечном счете, к сверхбыстрому локальному уплотнению. При этом вещество квазижидкой фазы выдавливается из уплотняющейся центральной области образца по направлению к его периферии. Вместе с ним перемещается область максимального поглощения микроволнового излучения, что приводит к насыщению тепловой неустойчивости во внутренней области, где уплотнение практически завершено. Таким образом, уплотнение распространяется от центра образца к его периферии в виде фронта, что приводит к быстрому формированию плотного керамического материала. Высокая и однородная конечная плотность образцов, а также однородный размер зерна в них [15] свидетельствуют о том, что квазижидкая фаза в ходе процесса спекания присутствует (возможно, в разные моменты времени) во всех областях объема образца.

Оценки, выполненные в соответствии с (4) для экспериментов с керамическими образцами $\mathrm{Yb}:\left(\mathrm{La}_{0.1} \mathrm{Y}_{0.9}\right){ }_{2} \mathrm{O}_{3}$ [15], показали, что возникновение тепловой неустойчивости происходит при значениях поглощаемой микроволновой мощности $p_{\text {abs }}$ в диапазоне от 15 до $400 \mathrm{~W} / \mathrm{cm}^{3}$ в зависимости от максимальной температуры нагрева образов. При этом напряженность электрического поля $E$ в образце изменяется в интервале 100-300 V/cm. Подобные значения поглощаемой микроволновой мощности $p_{\text {abs }}$ наблюдались также в процессах сверхбыстрого спекания образцов, компактированных из $\mathrm{Y}_{2} \mathrm{O}_{3}{ }^{-}$и $\mathrm{MgAl}_{2} \mathrm{O}_{4}$ порошков.

На наш взгляд, подобный механизм сверхбыстрого спекания также реализуется в процессаx flash sintering c приложением к образцам постоянного или низкочастотного переменного электрического напряжения. В таких процессах джоулев нагрев протекающим через образцы электрическим током также представляет собой объемный внутренний источник энергии в образце, в то время как отвод тепла от образца осуществляется через его поверхность. В работе [23] на основании анализа результатов, полученных различными авторами, показано, что характерные значения удельной плотности мощности, при которых наблюдается переход к сверхбыстрому спеканию широкого класса керамических материалов, лежат в интервале $10-50 \mathrm{~W} / \mathrm{cm}^{3}$ при напряженности электрического поля, варьируемой от 10 до $1000 \mathrm{~V} / \mathrm{cm}$. Сходство температурных профилей, возникающих при нагреве образцов микроволновым излучением и электрическим током, и близость характерных значений параметров, соответствующих развитию тепловой неустойчивости, позволяют полагать, что предложенный в настоящей работе механизм спекания объясняет также эффект сверхбыстрого спекания при приложении к образцам постоянного или низкочастотного переменного напряжения [5].

\section{Заключение}

Продемонстрировано сверхбыстрое микроволновое спекание оксидных керамических материалов $\mathrm{Y}_{2} \mathrm{O}_{3}$, $\mathrm{MgAl}_{2} \mathrm{O}_{4}$ и $\mathrm{Yb}:(\mathrm{LaY})_{2} \mathrm{O}_{3}$. Плотность более $98-99 \%$ от теоретического значения достигалась в указанных материалах за времена, измеряемые единицами минут, при отсутствии стадии изотермической выдержки. На основании анализа экспериментальных данных (мощность микроволнового излучения, скорости нагрева и остывания) и исследований микроструктуры полученных материалов предложен механизм сверхбыстрого спекания, основанный на размягчении и плавлении границ зерен.

Следует отметить, что с точки зрения практических приложений процесс высокоскоростного спекания с использованием микроволнового нагрева не требует применения электродов для обеспечения требуемого удельного вклада мощности в спекаемые изделия, что является очевидным преимуществом данного метода. Не менее важной представляется возможность эффективного управления процессом развития тепловой неустойчивости при использовании быстродействующих систем автоматического управления процессом нагрева.

Сверхбыстрое микроволновое спекание является новым методом, имеющим значительные преимущества с точки зрения потребления энергии и продолжительности процессов. Необходимы дальнейшие исследования, направленные на оптимизацию таких факторов, как свойства порошковых материалов, композиция, форма и размеры спекаемых изделий, физико-механические, функциональные и эксплуатационные свойства изделий, получаемых данным методом.

Исследование выполнено за счет гранта Российского научного фонда (проект № 17-19-01530).

\section{Список литературы}

[1] Munir Z.A., Quach D.V., Ohyanagi M. // J. Am. Ceram. Soc. 2011. Vol. 94. P. 1-19.

[2] Raj R., Cologna M., Francis J.S.C. // J. Am. Ceram. Soc. 2011. Vol. 94. P. 1941-1965. 
[3] Guillon O., Gonzales-Julian J., Dargatz B., Kessel T., Schierning G., Rathel J., Herrmann M. // Adv. Eng. Mater. 2014. Vol. 16. P. 830-849.

[4] Cologna M., Prette A.L.G., Raj R. // J. Am. Ceram. Soc. 2011. Vol. 94. P. 316-319.

[5] Yu M., Grasso S., McKinnon R., Saunders Th., Reece M. J. // Advances in Appl. Ceramics. 2017. Vol. 116. P. 24-60.

[6] Фок В.А. // Труды ЛФТИ. 1928. Т. 5. С. 52.

[7] Raj R. // J. Eur. Ceram. Soc. 2012. Vol. 32. P. 2293-2301.

[8] Roussy G., Bennani A., Thiebaut J.M. // J. Appl. Phys. 1987. Vol. 62. P. 1167-1170.

[9] Bykov Y.V., Rybakov K.I., Semenov V.E. // J. Phys. D: Appl. Phys. 2001. Vol. 34. P. R55-R75.

[10] Semenov V. E., Zharova N. A. // Advances in Microwave and Radio Frequency Processing (Proc. 8th International Conference on Microwave and High-Frequency Heating) / Ed. by M. Willert-Porada. Berlin: Springer, 2006. P. 482-490.

[11] Bykov Yu., Eremeev A., Glyavin M., Kholoptsev V., Luchinin A., Plotnikov I., Denisov G., Bogdashev A., Kalynova G., Semenov V., Zharova N. // IEEE Trans. Plasma. Sci. 2004. Vol. 32. P. 67-72.

[12] Kremer F., Izatt J.R. // Int. J. Infrared Milli. Waves. 1981. Vol. 2. P. 675-694.

[13] Kimrey H.D., Janney M.A. // Microwave Processing of Materials (Mater. Res. Soc. Symp. Proc. Vol. 124) / Ed. by W.H. Sutton, M.H. Brooks, I.J. Chabinsky. Pittsburgh: Materials Research Society, 1988. P. 367-372.

[14] Джексон Джс. Классическая электродинамика. М.: Мир, 1965.

[15] Bykov Yu.V., Egorov S.V., Eremeev A.G., Kholoptsev V.V., Rybakov K.I., Sorokin A.A. // J. Am. Ceram. Soc. 2015. Vol. 96. P. 3518-3524.

[16] Bykov Yu.V., Egorov S.V., Eremeev A.G., Kholoptsev V.V., Plotnikov I.V., Rybakov K.I., Sorokin A.A. // Materials. 2016. Vol. 9. P. 684.

[17] Li J.-G., Ikegami T., Lee J.-H., Mori T. // J. Am. Ceram. Soc. 2000. Vol. 83. P. 2866-2868.

[18] Hao Q., Li W., Zeng H., Yang Q., Dou Ch., Zhou H., Lu W. // Appl. Phys. Lett. 2008. Vol. 92. P. 211106

[19] German R.M. Sintering Theory and Practice. N.Y.: Wiley, 1996.

[20] Kochawattana S., Stevenson A., Lee S.-H., Ramirez M., Gopalan V., Dumm J., Castillo V.K., Quarles G.J., Messing. G.L. // J. Eur. Ceram. Soc. 2008. Vol. 28. P. 1527-1534.

[21] Johnson D.L. // J. Am. Ceram. Soc. 1991. Vol. 74. P. 849-850.

[22] Broughton J.O., Gilmer G.M. // J. Phys. Chem. 1987. Vol. 91. P. 6347-6359.

[23] Raj R. // J. Am. Ceram. Soc. 2016. Vol. 99. P. 3226-3232. 